\title{
Patrimonio histórico-minero en El Espinar (Segovia): primera aproximación al catálogo de bienes y elementos
}

\section{R. Díez Marcelo ${ }^{a}$, J.J. García Criado ${ }^{b}$ y A. Díez Herrero ${ }^{c}$}

${ }^{\mathrm{a} C}$ Colegio Claret Segovia, Avda. del Padre Claret 5, 40003 Segovia. ruydiezma@gmail.com, ${ }^{\mathrm{b} C E P S A ~ E . ~ P ., ~ S . A ., ~ T o r r e ~ C e p s a, ~ P a s e o ~}$ de la Castellana 259 A, 28046 Madrid. jose.garciac@cepsa.com y ${ }^{\mathrm{c} I n s t i t u t o ~ G e o l o ́ g i c o ~ y ~ M i n e r o ~ d e ~ E s p a n ̃ a, ~ C a l l e ~ R i ́ o s ~ R o s a s ~ 23, ~}$ 28003 Madrid. andres.diez@igme.es

\begin{abstract}
Resumen
En el presente estudio se recopilan todos los restos de actividad minera histórica, de especial importancia patrimonial, localizados en el término municipal de El Espinar (sector suroccidental de la provincia de Segovia, Castilla y León). La minería en este municipio ha sido de especial importancia desde, al menos, época romana; pero no es hasta mediados del siglo XIX cuando alcanza su mayor apogeo. En el primer y tercer cuartos del siglo XX, se reactiva la actividad minera y se realizan labores de especial interés patrimonial. Tras el abandono definitivo de la actividad minera en la década de 1960, se ha producido la destrucción de gran parte este patrimonio por motivos diversos. Derivado de toda esta actividad, se han localizado, documentado y catalogado más de un centenar de restos, entre los que predominan las zanjas y escombreras (más de medio centenar de cada una); pero también pozos, edificios, lavaderos y hasta un molino de mineral. En la última década han aparecido posibles figuras de protección que deberían ser los canales para conseguir el inventario sistemático, protección y divulgación de este patrimonio histórico-minero.
\end{abstract}

Palabras clave: minería, historia, patrimonio, catálogo, El Espinar, Segovia, Sierra de Guadarrama.

\begin{abstract}
This study compiles all the remains of historic mining activity of special heritage value in the municipality of El Espinar (Southwestern sector of the province of Segovia, Castilla y León, Central Spain). Mining in this municipality has been of special importance since, at least, Roman times; but it is not until the middle of the 19th Century that it reaches its peak. In the first and third quarters of the 20th Century, mining activity was reactivated and works of special heritage interest were carried out. After the permanent closure of mining activity in the 1960 decade, a great part of this heritage has been destroyed for various reasons. As a result of all this activity, more than a hundred remains have been located, documented and cataloged, among which ditches and dumps predominate (more than half a hundred of each one); but also wells, buildings, washing infrastructures and even a mineral mill. There are possible frameworks of protection that have appeared in the last decade and that should be the channels to obtain the systematic inventory, protection and dissemination of this historic-mining heritage.
\end{abstract}

Keywords: mining, history, heritage, catalogue, El Espinar, Segovia, Sierra de Guadarrama. 


\section{Introducción}

El estudio y conservación del patrimonio histórico-minero ha sido tradicionalmente relegado frente a la recuperación de otros patrimonios histórico-industriales con mayor componente arquitectónico, como el fabril (textil, agroalimentario, siderúrgico...) o el energético e infraestructural. Y dentro de aquel, el patrimonio minero situado fuera de los grandes núcleos mineros históricos (Riotinto, Almadén, La Unión...), ha pasado desapercibido, lo que ha llevado en múltiples ocasiones a su deterioro o desaparición por desconocimiento o desidia. Este es el caso del patrimonio histórico minero de la Sierra de Guadarrama y su entorno y, en particular, de los restos de labores mineras de la vertiente septentrional del Guadarrama en el término municipal de El Espinar (provincia de Segovia). Por lo anteriormente expuesto, el objetivo del presente trabajo es realizar un primer inventario de bienes y elementos histórico-mineros en el término municipal de El Espinar; contextualizándolos históricamente y haciendo patentes sus principales amenazas y oportunidades de futuro.

\section{Breve reseña histórica de la actividad minera en El Espinar}

Posiblemente la explotación de las mineralizaciones existentes en El Espinar se remonte al Neolítico, prolongándose siglos después durante la Edad del Hierro. Pero la explotación intensiva de los yacimientos minerales no comienza hasta bien entrada la Edad Antigua. Es posible que esta zona perteneciese al posible distrito minero romano (metalla) del Cerro de los Almadenes (San Clemente y Ayarzagüena, 2019), ya que existen indicios de la existencia de dos escoriales de posible origen romano (Pérez, 1920) en las inmediaciones de San Rafael (pedanía de El Espinar).

Dando un gran salto temporal, en los indicios de cobre y estaño del Reino de Castilla de los siglos XV y XVI, entre otros, se cita la minería en este municipio (Díez y Martín Duque, 2005).

Pero no es hasta el s. XIX con la concesión de las minas "San Quintín" y "Reina" y la apertura del molino cuando la actividad minera cobra importancia en la zona. En 1854, la Compañía Minera Los Carpetanos denuncia la mina "San Quintín". En esta concesión las únicas labores consistían en un pozo de 12,5 metros (Cortázar, 1891) que aún no se ha podido localizar por insuficiencia de datos acerca de su ubicación. Un año después, se denuncia, también en nombre de la Compañía Minera Los Carpetanos, la mina "Reina", cuyo beneficio sería el cobre. Las labores de "Reina" consistían en un pozo de 30 metros de profundidad con dos galerías en dirección que aún se conserva junto al molino que se habilitó para procesar el mineral de estas dos minas.

Tras casi el completo abandono de la actividad minera, a principios del s. XX se descubre un potente filón de cuarzo con torbernita (fosfato de uranio y cobre, mineral radiactivo de la familia de las 'micas del uranio') en la mina "Flor del Espinar" (Estadística Minera, 1905). Las labores de esta concesión consistían en dos pozos de seis metros de profundidad y varias calicatas (Estadística Minera, 1909). En 1909 las minas se encontraban inundadas y ya no se trabajaba en ellas.

Ocho años después, en 1917, el Ingeniero Jefe del Distrito de Madrid, D. Pedro Pérez, realiza el "Estudio de los Criaderos Minerales de San Rafael (Segovia)" que sería publicado tres años después (Pérez, 1920). Sin el trabajo realizado por Pedro Pérez habría sido imposible localizar y conocer el nombre de la mayoría de las labores mineras existentes en el término municipal, algunas de las cuales han sido destruidas. Es el caso del pozo Cacera, situado en la margen izquierda del río Gudillos y que fue destruido con la apertura de la vía de servicio de la AP-6. En octubre de 1905 en el pozo de la Cacera se había profundizado 14 metros (Fernández, 1905). Según Pedro Pérez se trataba de un pozo de 2,5 metros de sección por 21 metros de profundidad con dos galerías enfrentadas en el fondo, de dirección noroeste y sureste, una de tres metros y otra de doce. A los 9 metros de profundidad el propio Pedro Pérez realizó una galería cuyas labores se interrumpieron por las filtraciones del río y otra interrumpida a los $8,8 \mathrm{~m}$ por estrecharse el filón. Según Pedro Pérez el pozo de la Cacera se encontraba continuamente desaguado por un torno y protegido por un chozo (Pérez, 1920) que, según atestigua Juan José Palomero (vecino de San Rafael), se conservó hasta los años 30-40 del siglo XX.

Para 1923 cesa la demarcación de minas en El Espinar, y no es hasta 1942, con la llegada a El Espinar de Gregorio y Alberto Criado, cuando se retoma. Estos se centran casi exclusivamente en la revisión y reapertura de los criaderos del 
cerro de Cabeza Reina, denunciando la mina "El Carmen" (1944); aunque con excepciones, como la nueva apertura de las minas "Magda" y "Ampliación a Magda" (1959) de wolframio y estaño en El Estepar. Más o menos al mismo tiempo, llega a El Espinar una empresa salmantina que se centra fundamentalmente en las labores del cerro de El Estepar y denuncia la mina "Julita".

En 1951 según la Estadística Minera "tan solo se realizan trabajos consistentes en rafas o calicatas de escasa profundidad y desarrollo sobre afloramientos" (Estadística Minera, 1951). En el año que le sigue (1952) tan solo se realizó en San Rafael “...un pozo de 12 metros de profundidad y galería al nivel 10, de 16 metros de longitud...” en la mina "Esperanza” (Estadística Minera, 1952).

Durante las décadas de 1950 y 1960 se realizaron labores de prospección e investigación en otros lugares del término municipal de El Espinar sin que dieran lugar a labores y explotaciones reseñables.

A partir de inicios de la década de 1960, en El Espinar tan solo se denuncian concesiones sin labores documentadas y la minería en la zona comienza a sufrir una clara decadencia que lleva al abandono por completo de las labores y, en algunos casos, su destrucción.

\section{Catálogo de bienes y elementos de interés patrimonial}

Como fruto de esa dilatada pero resumida historia del apartado anterior, se han conservado restos de las labores mineras en el término municipal que se concentran fundamentalmente en cuatro sectores del territorio (Fig. 1). Los restos de actividades mineras son tanto de carácter superficial (zanjas, calicatas y rafas) como subterráneo (galerías y pozos de pequeñas dimensiones y longitud). Algunas de estas labores (las de mayor entidad) vienen acompañadas de instalaciones anexas (lavaderos, casetas de acopio de mineral y herramientas...).

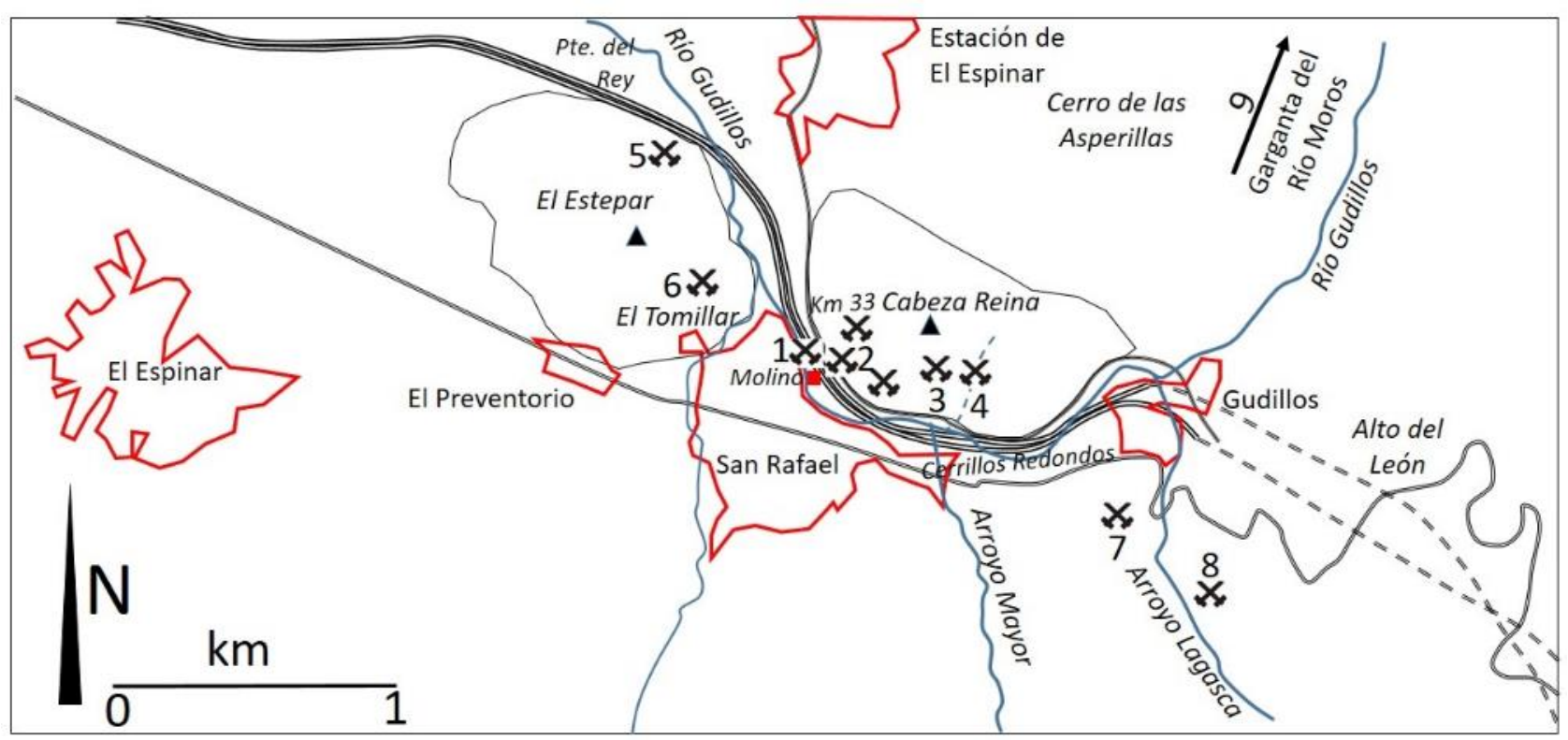

Fig. 1 Mapa esquemático de la situación de los principales restos de patrimonio histórico-minero de El Espinar. Obsérvese que en su mayoría se encuentran en torno al núcleo de población de San Rafael

La tabla 1 recoge el número de elementos patrimoniales de diferentes tipos presentes en cada uno de los principales grupos mineros del término municipal de El Espinar.

\subsection{Grupo del cerro de Cabeza Reina}

El cerro de Cabeza Reina (1479 m.s.n.m.) se localiza al noroeste de San Rafael (Fig. 1). En este cerro se localizan las labores de la mina "El Carmen" (1944) y los restos de la mina "Reina" (1855) en el fondo del valle, junto al río Gudillos; además de algunas labores mineras fuera del contexto del cerro, como es el caso de la "Calicata de la Caseta". 
Molino del río Gudillos y pozo de la mina "Reina"

En las proximidades de la localidad de San Rafael y más concretamente en la margen izquierda del río Gudillos (número 1 en la figura 1), se conservan las ruinas de un antiguo molino (Fig. 2) con la función de moler el mineral de las minas "Reina" y "San Quintín", que fue abierto aproximadamente en el año 1860 por la Sociedad Minera Los Carpetanos (De Bustos, 1860). Consistía en "un bocarte de dos baterías y dos mesas de percusión, movido todo por una rueda de cajones" (Cortázar, 1891). En las proximidades del molino, entre abundante vegetación arbustiva, se encuentra el pozo de la mina "Reina". Se trata de un pozo circular de dos o tres metros de diámetro, recubierto de ladrillo y parcialmente obstruido por unas viguetas de hormigón que seguramente taparían el pozo entero para evitar que cayera el ganado, tras su abandono.

Tabla 1. Elementos de principal interés patrimonial histórico-minero del término municipal de El Espinar. Leyenda de abreviaturas: Ca, Calicatas, trincheras, zanjas o rafas; Es, Escombrera; Ed, Edificación; Ma, Maquinaria; La, Lavadero o balsas; Mo, Molinos; Ac, Acceso y extracción; Ve, Ventilación; GH, Galerías horizontales; *, se trata del mismo elemento

\begin{tabular}{|c|c|c|c|c|c|c|c|c|c|c|c|}
\hline \multirow{4}{*}{$\begin{array}{l}\text { PARAJE O } \\
\text { CERRO }\end{array}$} & \multirow{4}{*}{\multicolumn{2}{|c|}{ LABOR / CONCESIÓN }} & \multicolumn{9}{|c|}{$\begin{array}{c}\text { ELEMENTOS O BIENES DE INTERÉS PATRIMONIAL } \\
\text { HISTÓRICO-MINERO }\end{array}$} \\
\hline & & & \multicolumn{6}{|c|}{$\begin{array}{c}\text { Superficiales (sobre la superficie del } \\
\text { terreno) }\end{array}$} & \multicolumn{3}{|c|}{ Subterráneos } \\
\hline & & & \multicolumn{2}{|c|}{$\begin{array}{l}\text { Labores } \\
\text { mineras }\end{array}$} & \multicolumn{4}{|c|}{$\begin{array}{c}\text { Instalaciones anexas } 0 \\
\text { complementarias }\end{array}$} & \multicolumn{2}{|c|}{ Pozos } & \multirow[t]{2}{*}{ GH } \\
\hline & & & $\mathbf{C a}$ & Es & Ed & Ma & La & Mo & Ac & Ve & \\
\hline \multirow{7}{*}{$\begin{array}{c}\text { CERRO } \\
\text { CABEZA } \\
\text { REINA }\end{array}$} & & Arroyo Majada Alta & 1 & 1 & - & - & - & - & - & - & 1 \\
\hline & Mina "El Carmen" & Kilómetro 33 & - & 3 & 2 & $1 *$ & 1 & $1 *$ & - & - & - \\
\hline & & Galería con puntal & 1 & 1 & - & - & - & - & - & - & 1 \\
\hline & Calicata de la Caseta & & 1 & 1 & - & - & - & - & - & - & 1 \\
\hline & Calicata "Santa Rosa' & & 1 & 1 & - & - & - & - & - & - & - \\
\hline & Mina "Luis" & & - & 1 & - & - & - & - & 1 & - & - \\
\hline & Mina "Reina" & & - & 2 & - & - & - & 1 & 1 & - & - \\
\hline \multirow{3}{*}{$\begin{array}{l}\text { CERRO EL } \\
\text { ESTEPAR }\end{array}$} & Pozo “Ángel” & & - & 1 & - & - & - & - & 1 & 1 & - \\
\hline & Paraje de 'Barrera del & Toro' & 1 & - & - & - & - & - & - & - & - \\
\hline & Minas "Julita", "Mag & a" y “Ampliación a Magda" & $>30$ & $>30$ & 2 & - & 1 & - & - & - & 1 \\
\hline LA GASCA & Minas "Amable" y " $\mathrm{T}$ & rio" & $>20$ & $>20$ & - & - & - & - & - & - & - \\
\hline \multirow[t]{2}{*}{$\begin{array}{l}\text { GARGANTA } \\
\text { DEL RÍO }\end{array}$} & Mina "San Juan de Di & s" o "Mina Bermeja" & - & 1 & - & - & - & - & - & - & 1 \\
\hline & & TOTAL (número) & $>55$ & $>62$ & 4 & 1 & 2 & 2 & & & 5 \\
\hline
\end{tabular}

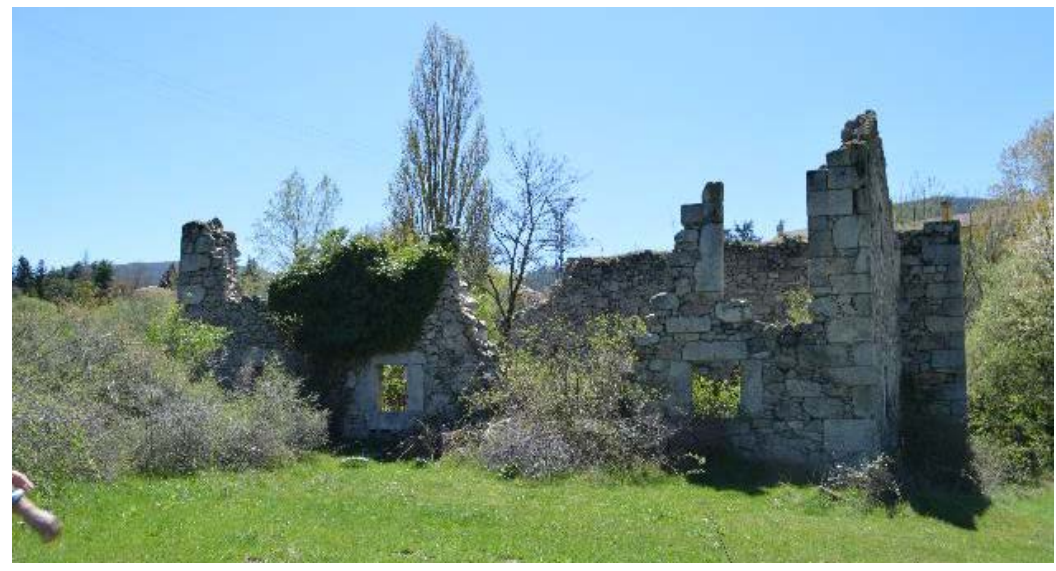

Fig. 2 Molino de mineral del río Gudillos, donde se aprecia su estado de deterioro actual 


\subsubsection{Labores e instalaciones de la mina "El Carmen"}

En el cerro de Cabeza Reina se encuentran diseminadas las labores de la mina "El Carmen", denunciada en el año 1944. La mayoría de labores ya se encontraban realizadas previamente y tan solo se hicieron a mediados del siglo XX, algunos trabajos de ampliación y aprovechamientos 'de rapiña'. Las labores de mayor importancia de esta mina son las existentes en el km. 33 de la vía férrea Villalba-Segovia (Fig. 1). Actualmente solo se conservan sus escombreras arrasadas por una pista forestal, un piñón de hierro del engranaje de un molino de los años 40 habilitado para machacar el mineral de la mina (Fig. 3) y las ruinas de dos casetas en las que vivían los mineros y se guardaban las herramientas.

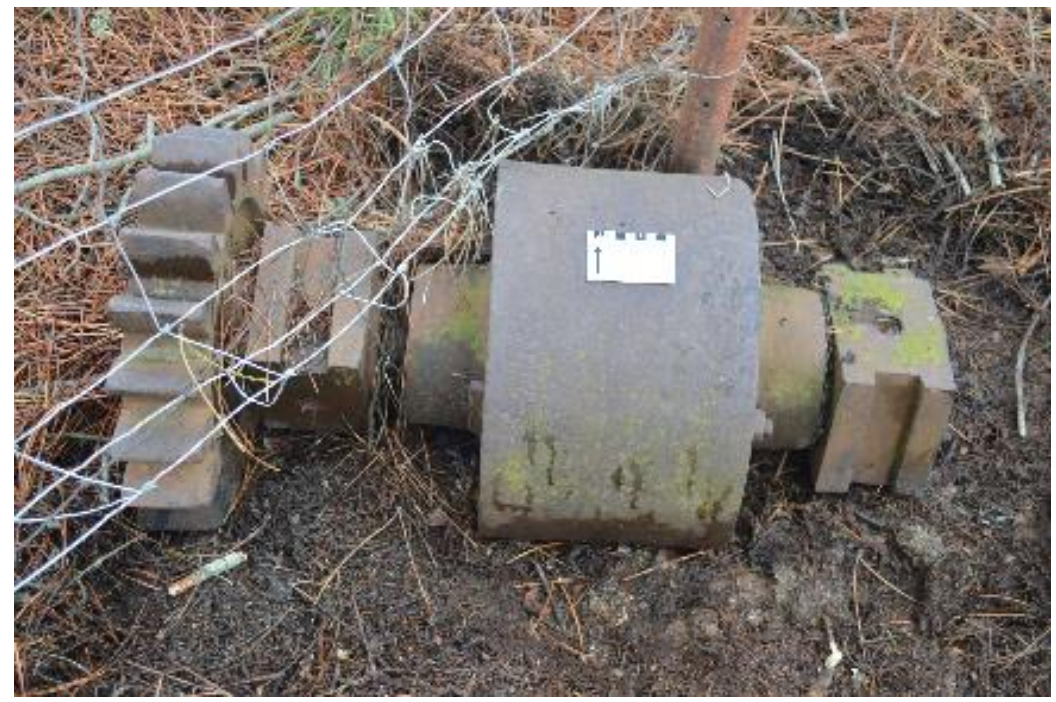

Fig. 3 Piñón metálico del engranaje de un molino de mineral en el km. 33 de la vía férrea

También hay más labores de interés en esta mina, como pueden ser la pequeña galería existente en las cercanías del túnel del ferrocarril que contiene un madero de sujeción en el techo, a modo de puntal (número 2 en la figura 1); o la galería y el pozo que se encuentran en las inmediaciones del arroyo de Majada Alta, al este del cerro (números 3 y 4 en la figura 1) que pertenecieron anteriormente a la concesión "Luis" (1906). Esta galería tiene 11 metros de longitud, mientras que el pozo se encuentra cegado casi hasta su borde.

\subsection{Grupo de labores del cerro de El Estepar}

El cerro de El Estepar (1346 m.s.n.m) se encuentra al noroeste de San Rafael y al noreste de El Espinar (Fig. 1). En este cerro se pueden encontrar gran cantidad de zanjas y una galería, que constituyen las labores de las minas "Julita" y "Magda", además de dos pozos (pozo Ángel y su pozo de ventilación) de principios del siglo XX.
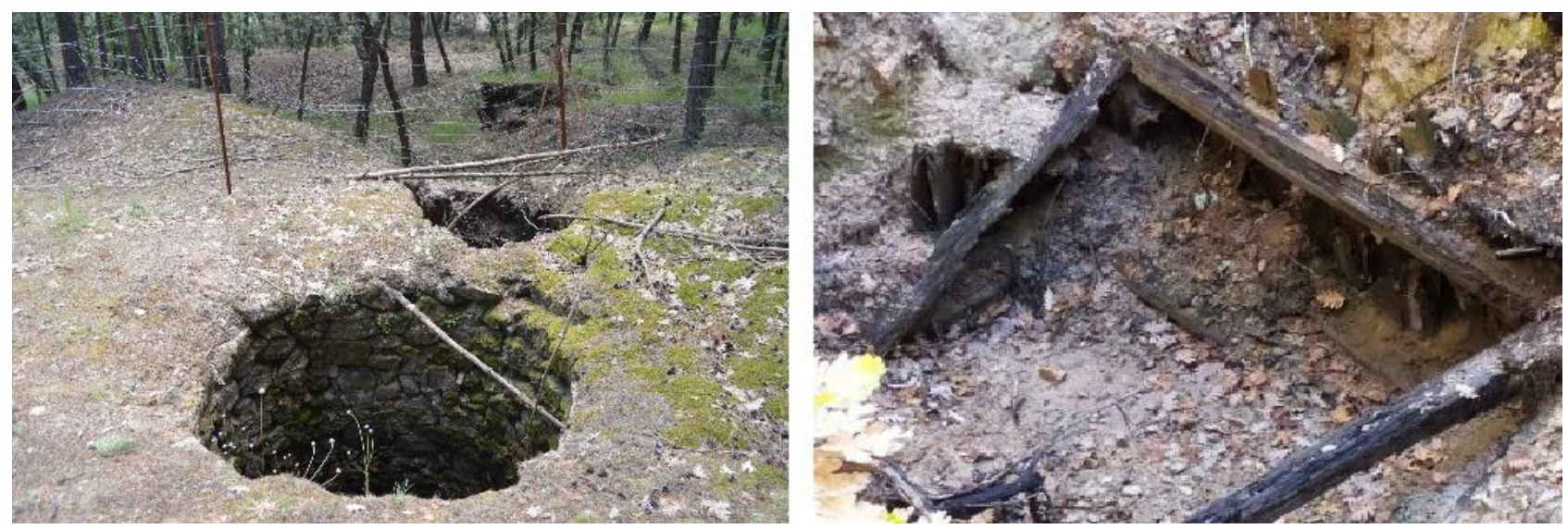

Fig. 4 Izquierda, pozo mamposteado de ventilación del pozo Ángel. Derecha, aspecto actual del pozo Ángel. Como se puede observar, a pesar de encontrarse colmatado con tierra hasta su borde, se conserva la antigua entibación de madera. Obsérvese que las galerías que unen los dos pozos están colapsando 


\subsubsection{Pozo Ángel y pozo de ventilación}

En la ladera noreste del cerro de El Estepar se localizan dos pozos abiertos a principios del s. XX (número 5 en la figura 1). Uno de ellos está colapsando y se encuentra inundado la mayor parte del año. En los meses secos, se advierte la estructura de entibado de madera que recubría el pozo, que llegó a tener 30 metros de profundidad y dos galerías en dirección en su interior (Pérez, 1921). El otro pozo, que hacía las veces de respiradero para ventilación, se encuentra mamposteado y en su fondo se advierte la existencia de una galería en dirección (Fig. 4).

\subsubsection{Labores e instalaciones de las minas "Julita" y "Magda"}

En la ladera este del cerro de El Estepar se conservan las labores de mayor entidad en el término municipal, que fueron realizadas dentro de las concesiones "Julita" y "Magda" durante los las décadas de 1950 y 1960 para la extracción de wólfram (número 6 en la figura 1). Las principales labores consisten en una galería de 20 metros de profundidad y gran cantidad de zanjas y calicatas diseminadas por todo el cerro, alineadas siguiendo las direcciones de los principales haces filonianos de cuarzo mineralizado. Entre las instalaciones destacan dos casetas para acopio de herramientas y mineral y una alberca en un arroyo para lavar el mineral de las minas (Fig. 5). Una de estas dos casetas pudiera corresponder con la “...caseta de mampostería de dos metros de lado ubicada en la mina Magda para almacén de herramientas y alojamiento del guarda...” que se autorizó construir en 1955 (Ceballos y Jiménez, 2001).

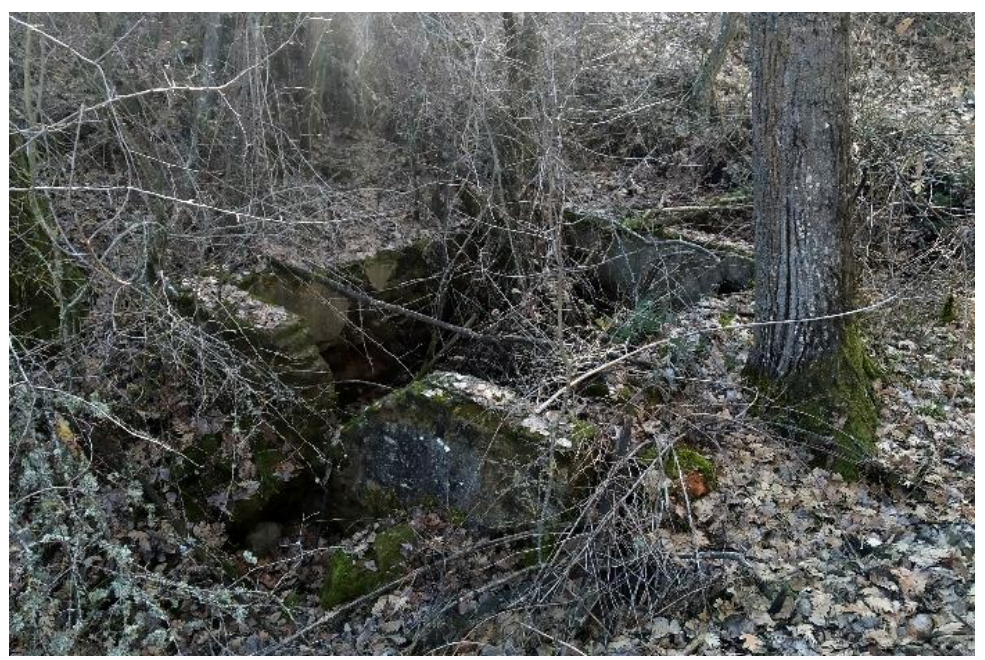

Fig. 5 Alberca existente en El Estepar, construida para lavar el wolframio de las minas durante la década de 1950

\subsection{Grupo de labores de La Gasca}

En la ladera norte de Cabeza Líjar, junto a las curvas de la carretera N-VI en el acceso septentrional al puerto del Alto del León (1510 m.s.n.m) y más concretamente en los parajes de El Umbrión, Los Talleres y La Gasca, hay dos pequeñas agrupaciones de zanjas y calicatas de pequeñas dimensiones que fueron realizadas en el primer cuarto del siglo XX en las concesiones "Amable" y "Torio" (números 7 y 8 en la figura 1).

\subsection{Grupo de labores de La Garganta del río Moros}

A $8 \mathrm{~km}$ de San Rafael, en la Sierra de Quintanar y más concretamente en la ladera este del Cerro Puerco, en la margen izquierda del arroyo Blasco-Malo se encuentra el paraje de "La Mina". Posiblemente este paraje anteriormente se denominaba "Dehesa de la Bermeja", por pertenecer a tierras de los Bermejo en los siglos XV y XVI (Rodríguez Arce, 1916). En 1860 D. José Sáenz Tejada denuncia la mina "San Juan de Dios" en la Dehesa Bermeja, aunque se sabe que la había estado explotando desde 1859 (A.M.S., 1245-3). En esta época se realizó un pozo, junto al que el 12 de octubre de 1859 se solicitó realizar una caseta que sirviera de albergue para los mineros (A.M.S., 1245-3). Tras esta actividad minera, el paraje pasó a denominarse "La Mina Bermeja". En 1916 se denuncia la "Mina Bermeja" en el paraje del mismo nombre (Larrondo, 1916); y en esta época se debió de realizar la galería que es visible hoy en día. Con los años, el topónimo de "La Mina Bermeja" derivaría en "La Mina", tal y como aparece representada en la cartografía moderna. 


\section{Principales amenazas y oportunidades para el patrimonio}

Tras el abandono de la actividad minera, durante las décadas de 1970 a 1990, se produce la destrucción de gran parte del patrimonio histórico-minero por diferentes motivos, entre los que cabe destacar el trazado de infraestructuras, los procesos urbanísticos y las actividades forestales y ganaderas (Fig. 6).
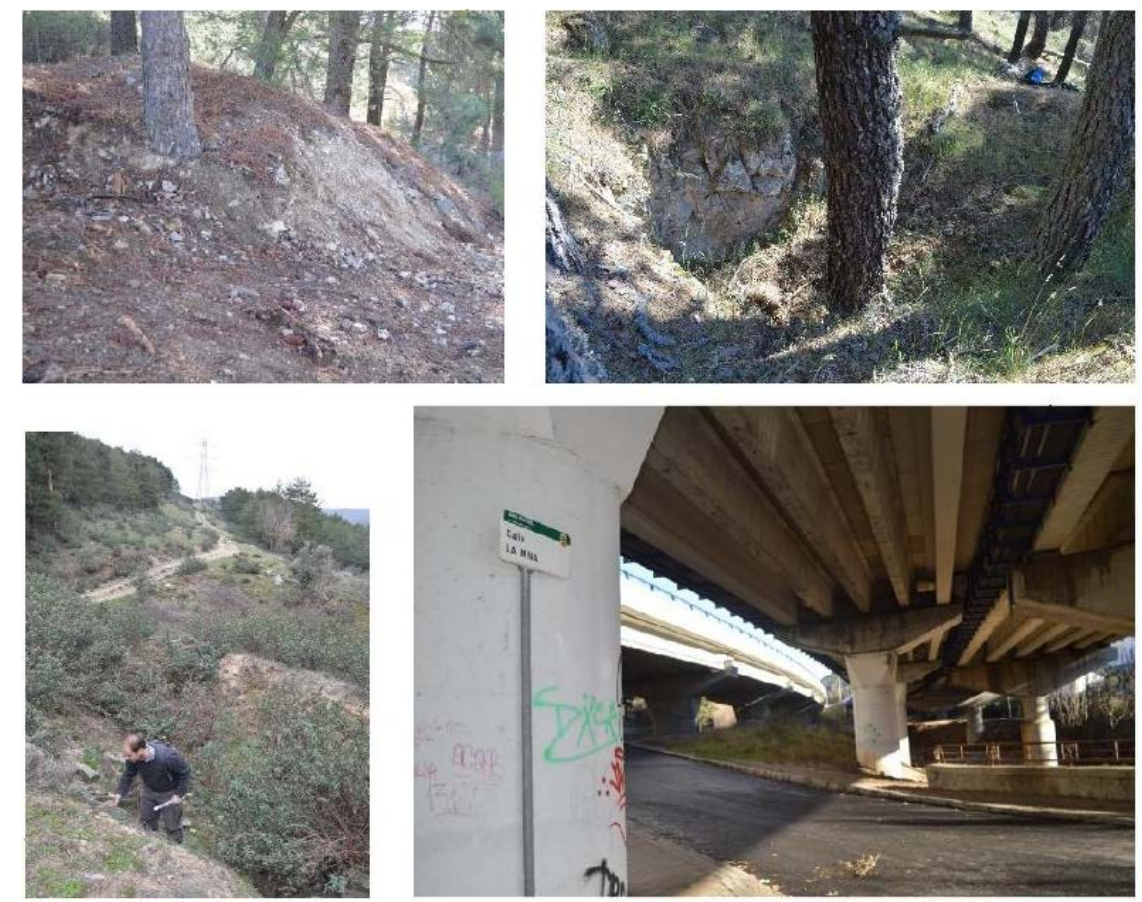

Fig. 6 Arriba a la izquierda, escombreras de las labores de la mina "El Carmen" situadas en el km. 33 afectadas por la apertura de una pista forestal siguiendo la vía férrea. Arriba a la derecha, pozo de la mina "Luis"obstruido por las labores de aterrazamiento. Abajo a la izquierda, aspecto de la "Calicata Santa Rosa" afectada por la apertura del cortafuegos de Cabeza Reina. Y abajo a la izquierda, actual calle La Mina donde se encontraban las labores de "Flor del Espinar"

El trazado y ampliación de infraestructuras comprende tanto el transporte de energía como de personas y mercancías. En el primer caso se trata fundamentalmente de tendidos eléctricos ( $\mathrm{y}$ en menor medida gasoductos), cuyo movimiento de tierras ha degradado labores mineras históricas como la "Calicata Santa Rosa" en la ladera suroeste del cerro de Cabeza Reina o varias zanjas existentes en el cortafuegos de El Espinar; además de las zanjas y calicatas existentes en la concesión "Flor de El Espinar" sobre el túnel del ferrocarril en Cabeza Reina. Por su parte, la propia construcción del ferrocarril Villalba-Segovia produjo la destrucción de algunas labores en Cabeza Reina, a la vez que sirvió para descubrir otras. Pero sin duda la actividad más destructiva fue la ampliación de carreteras nacionales y comarcales, así como la construcción de las autopistas AP-61 y AP-61 y sus túneles.

Con la ampliación de la AP-6 fueron destruidas la mayor parte de las labores de la mina "Flor del Espinar" que se situaban junto a la actual calle La Mina, enfrente del apeadero de San Rafael; aunque la causa de su destrucción, como más tarde se apuntará, no se limitó a la ampliación de la AP-6. Además de las labores de la mina "Flor del Espinar", el pozo Cacera fue destruido con la construcción de una vía de servicio para la autopista en el acceso a Gudillos.

La urbanización de determinadas zonas colindantes a San Rafael ha provocado la destrucción de algunos elementos de patrimonio histórico minero, como pudo ser el escorial situado al sur del pozo Cacera, que se encontraría bajo la urbanización de Cerrillos Redondos; o las labores de la mina "Flor del Espinar", que fueron destruidas tanto por la ampliación de la AP-6 como con la construcción de varios chalets.

Respecto al efecto de las labores forestales y ganaderas, el aterrazamiento para reforestación (en el antiguo pozo de la mina "Luis" y Calicata de la Caseta), la apertura de cortafuegos ("Calicata Santa Rosa"), la apertura y mantenimiento de pistas forestales (labores de "El Carmen" en el km. 33 de la vía férrea), la nivelación y el movimiento de tierras tras incendios (de nuevo en el pozo de la concesión "Luis" y en la Calicata de la Caseta, además de multitud de labores de 
El Estepar) y otras labores en el monte para su uso ganadero (cegamiento de pozos para evitar accidentes de ganado y personas, y utilización de zanjas y pozos como abrevaderos y albercas) han hecho desparecer también parte de este patrimonio minero o lo han deteriorado.

En la última década, figuras de protección como el parque natural de la Sierra de Guadarrama Norte o la reserva de la biosfera del Real Sitio de San Ildefonso-El Espinar, deberían ser los canales para conseguir el inventario, protección y divulgación de este patrimonio histórico-minero. También el planeamiento urbanístico del municipio de El Espinar debería incluir estos elementos en el catálogo de elementos culturales de interés patrimonial, y estos sectores como suelo rústico no urbanizable de especial protección cultural.

\section{Conclusiones}

El municipio de El Espinar tiene una larga y rica historia de actividad minera. Esta ha dejado un variado patrimonio minero formado por más de un centenar de restos de labores mineras (pozos, zanjas, calicatas, rafas, galerías...) y sus instalaciones (casas de acopio de mineral y herramientas, molinos de machaqueo, lavaderos...). Existen múltiples amenazas para estos restos de patrimonio histórico-minero, bien por el trazado y ampliación de infraestructuras, la urbanización de áreas colindantes a San Rafael y las labores forestales y ganaderas. En la actualidad se divisan posibles acciones de conservación y recuperación de ese patrimonio a través de figuras de protección y el planeamiento territorial.

\section{Agradecimientos}

En primer lugar, gracias a Guadalupe de Marcelo e Isabel Álvarez por colaborar en la búsqueda documental de información histórica asociada a las labores mineras. Especial agradecimiento a: Julia Alonso, Juan José Palomero y Victoria Navas por haber accedido a ser entrevistados y habernos proporcionado información sobre la localización de algunas labores mineras; Luis Jordá Bordehore, Manuel Regueiro, Isabel Rábano, Iván Aguilera Díez y Ester Boixereu Vila, por apoyar el proyecto y haber aportado información acerca del paradero de algunos documentos; a Jorge Sanz, Alberto Rodríguez y Esteban Calvo por mostrarnos la localización de algunas labores; y por último a Javier Plaza por velar por la protección de este rico patrimonio minero.

\section{Referencias}

Archivo Municipal de Segovia. Doc. 1245-3. Expediente de solicitud de apertura de una caseta en la mina «San Juan de Dios». (9 de marzo de 1859-24 de noviembre de 1859).

Ceballos Aranda, J., y Jiménez Fernandez, F. J. (2001). Revisión de ordenación del grupo núm. 13 de la provincia de Segovia: Aguas Vertientes y agregados ( $8^{\circ}$ periodo de ordenación). L. I memoria, Junta de Castilla. Consejería de medio natural. Servicio territorial de Segovia. Unidad de ordenación y mejora. Documento inédito.

De Bustos Castilla-Portugal, R. (1860, 17 de marzo). Sin título. Gaceta de Madrid.

Díez Herrero, A., y Martín Duque, J. F. (2005). Las raíces del paisaje: Condicionantes geológicos del territorio de Segovia. Salamanca: Junta de Castilla y León.

Fernández Navarro, L. (1905). Las minas de El Espinar. Boletín de la Real Sociedad Española de Historia Natural, V, $511-516$.

Larrondo Oquendo, A. (1916. 29 de septiembre). Secretaría-Negociado de Fomento-Minas-núm. 364. En Boletín Oficial de la provincia de Segovia, 1.

Ministerio de Fomento (1865-1952). Estadística Minera de España. Dirección General de Agricultura, Minas y Montes.

Pérez Sánchez, P. (1920). Estudio de los criaderos minerales de San Rafael (Segovia). Boletín Oficial de Minas y Metalurgia, IV (3233), 1-25.

Rodríguez Arce, D. (1916). Historia de la Ilustre Villa de El Espinar. Segovia: Imprenta de El Adelantado. 
San Clemente Geijo, P., y Ayarzagüena Sanz, M. (2019). El cerro de los Almadenes: metalla romana en la provincia de Segovia. En La minería y metalurgia históricas en el sudoeste europeo, La minería y la metalurgia del centro de la península ibérica a través de la historia (pp. 133-150). 